\title{
Characterization of the NEPOMUC primary and remoderated positron beams at different energies
}

J. Stanja ${ }^{\mathrm{a}, *}$, U. Hergenhahn ${ }^{\mathrm{b}}$, H. Niemann $^{\mathrm{b}}$, N. Paschkowski ${ }^{\mathrm{b}}$, T. Sunn Pedersen $^{\mathrm{b}}$, H. Saitoh ${ }^{\mathrm{a}, \mathrm{c}}$, E. V. Stenson ${ }^{\mathrm{b}}$, M. R. Stoneking ${ }^{\mathrm{d}}$, C. Hugenschmidt ${ }^{\mathrm{e}}$, C. Piochacz

\author{
${ }^{a}$ Max-Planck-Institut für Plasmaphysik, Boltzmannstr. 2, 85748 Garching, Germany \\ ${ }^{b}$ Max-Planck-Institut für Plasmaphysik, Wendelsteinstr. 1, 17491 Greifswald, Germany \\ ${ }^{c}$ Department of Advanced Energy, The University of Tokyo, Kashiwa 277-8561, Japan \\ ${ }^{d}$ Department of Physics, Lawrence University, Appleton, WI, 54911, USA \\ ${ }^{e}$ Heinz Maier-Leibnitz Zentrum (MLZ) and Physik Department E21, Technische Universität \\ München, Lichtenbergstr. 1, 85747 Garching, Germany
}

\begin{abstract}
We report on the characterization of the positron beam provided at the open beam port of the NEPOMUC facility at the Heinz Maier-Leibnitz Zentrum (MLZ) Garching. The absolute positron flux of the primary beam at $400 \mathrm{eV}$ and $1 \mathrm{keV}$ kinetic energy and of the remoderated beam at 5, 12 and $22 \mathrm{eV}$ were determined. Energy-dependent intensities in the range of $(1-5) \cdot 10^{8} \mathrm{e}^{+} / \mathrm{s}$ and $(2-6) \cdot 10^{7} e^{+} / \mathrm{s}$ have been observed for the primary and remoderated beam, respectively. We attribute the significant losses for the primary beam, in comparison with the expected value, to the non-adiabatic positron guiding in the beam line. We also measured the longitudinal energy distribution of the remoderated beam, yielding an energy spread below $3.3 \mathrm{eV}$. The mean transverse energy of the remoderated beam, determined from measurements in different final magnetic fields, was found to be below $1.3 \mathrm{eV}$. These results are likely to apply to the NEPOMUC beam delivered to other user stations.
\end{abstract}

Keywords: Positron beam; NEPOMUC; Beam intensity; Energy distribution

\section{Introduction}

Experiments using positrons are of broad, interdisciplinary interest for example in materials science [1], atomic and molecular physics [2], positronium (Ps) physics [3], fundamental research on matter-antimatter symmetry $[4,5]$ and surface physics [6]. Another novel topic is the creation of an electronpositron plasma in the laboratory, as suggested by some of the current authors $[7,8]$. Many of these projects benefit from the use of high-flux, high-brightness

\footnotetext{
* Corresponding author

Email address: juliane.stanja@ipp.mpg.de (J. Stanja )
} 
positron beams where the beam brightness is defined as flux normalized to the product of the beam diameter squared and the perpendicular energy [9]. One of the most intense sources of low-energy positrons worldwide is the NEutroninduced POsitron source MUniCh (NEPOMUC) [9, 10], which is part of the FRM II research reactor in Garching, Germany. Here, high-energy $\gamma$-rays produced from neutron-capture in ${ }^{113} \mathrm{Cd}$ generate positrons by pair production in $\mathrm{Pt}$ foils. After moderation in $\mathrm{Pt}$, the positrons are accelerated by electric fields and magnetically guided to the experiments. This beam, here referred to as the primary beam, is characterized close to the source by an intensity of $1.1 \cdot 10^{9} e+/ \mathrm{s}[11]$ and typically has energies up to $1 \mathrm{keV}$. This energy is determined by the electric acceleration potential. Optionally, a brightness-enhanced positron beam, hereafter referred to as the remoderated beam, can be produced by remoderation of the primary beam using a W single crystal in reflection geometry [12]. Its energy can be selected by biasing the W crystal appropriately and typically is on the order of $10 \mathrm{eV}$.

In preparation for our pair-plasma experiments, a characterization of both beams in terms of their intensity, spatial extent and energy distribution is crucial. In Ref. [11] two characteristics of the primary beam at $540 \mathrm{eV}$ and of the remoderated beam at $20 \mathrm{eV}$ were presented. However, they were determined at a location close to the source after which the beam is magnetically transported via several bends to the open beam port. Here we report on a characterization of the NEPOMUC beam at various energies as it arrives at the position of user experiments. The measurements were performed using a dedicated target chamber and a retarding field analyzer.

\section{Experimental methods}

\subsection{Beam profile}

We employ a method comparable to the knife-edge method [13] in order to determine the beam profile. A target plate is translated transversally through the beam, and the beam intensity collected on this target is recorded with respect to the target position. Considering the measurement in one dimension, there are the two limiting cases: (i) when the target entirely covers the beam, the total beam intensity is collected and (ii) when the target does not intersect the beam, no intensity is collected. Thus, by identifying case (i) with $x=0$ and assuming a Gaussian beam profile, the measured intensity with translation in one direction can be described by

$$
I(x)=I_{0}\left(1-\operatorname{erf}\left(\frac{x-x_{m}}{\sqrt{2} \sigma_{x}}\right)\right)
$$

with $x_{m}$ the center of the beam and $\sigma_{x}$ the root-mean-squared spread of the one-dimensional beam projection. Applying this method to two perpendicular axes gives the maximum area covered by the beam in the measurement plane. 


\subsection{Energy distribution}

Magnetically guided charged particles exhibit a motion that can be decomposed into the cyclotron motion and the motion along the magnetic guiding field. At a given position, a mean parallel energy $\left\langle E_{\|}\right\rangle$and a mean transverse energy $\left\langle E_{\perp}\right\rangle$ can be assigned to the ensemble. In the following, the methods to determine $\left\langle E_{\|}\right\rangle$and $\left\langle E_{\perp}\right\rangle$ are outlined. A more detailed description can be found in the literature [14].

\subsubsection{Measurement of the longitudinal energy distribution}

The measurement of the longitudinal energy component, using the retarding field technique, is straightforward. A potential barrier of height $V_{R}$ acts as a filter allowing only particles with $E_{\|}>V_{R}$ to pass. Particles with $E_{\|}<V_{R}$ are reflected. By varying $V_{R}$ the energy distribution of the beam can be sampled. The current associated with particles crossing the barrier can be expressed as a function of retarding potential by

$$
I\left(V_{R}\right) \sim \int_{V_{R}}^{\infty} f\left(E_{\|}\right) d E_{\|}
$$

where $f\left(E_{\|}\right)$denotes the distribution of the parallel beam energy within the ensemble of particles. From the collected signal the mean longitudinal energy $\left\langle E_{\|}\right\rangle$ as well as the longitudinal energy spread $\Delta E_{\|}$can be determined by comparison to a parametrized form of the cumulative energy distribution function. Alternatively, the derivative of Eq. (2) with respect to $V_{R}$ yields the energy distribution itself, from which one can obtain $\left\langle E_{\|}\right\rangle$and $\Delta E_{\|}$without prior assumptions.

Since our data for the remoderated beam were found to be adequately described by a Gaussian energy distribution, the collected signal is given by

$$
I\left(V_{R}\right) \sim\left(1-\operatorname{erf}\left(2 \sqrt{\ln 2} \frac{V_{R}-\left\langle E_{\|}\right\rangle}{\Delta E_{\|}}\right)\right) .
$$

\subsubsection{Measurement of the mean transverse energy}

For the measurement of the transverse energy component, we make use of the fact that in an adiabatically varying magnetic guiding field, 'adiabatic invariants' of the particle ensemble can be constructed. In particular, we use the invariance of

$$
\frac{\left\langle E_{\perp}\right\rangle}{B}
$$

where $\left\langle E_{\perp}\right\rangle$ denotes the mean transverse energy of the beam and $B$ the magnetic field at the position of the particles. From energy conservation it then follows that an increase in parallel energy must be compensated by a decrease in perpendicular energy and vice versa (if no work is performed on the particle beam). Thus, in a magnetic field that is changing adiabatically from the initial value $B_{i}$ to the final value $B_{f}$, the mean longitudinal energy in the final field $\left\langle E_{\|, f}\right\rangle$ can be expressed by

$$
\left\langle E_{\|, f}\right\rangle=\left\langle E_{\|, i}\right\rangle+(1-\alpha)\left\langle E_{\perp, i}\right\rangle
$$


with $\alpha=B_{f} / B_{i}$ and $\left\langle E_{\perp, i}\right\rangle$ and $\left\langle E_{\|, i}\right\rangle$ the initial mean transverse and longitudinal energy, respectively. $\left\langle E_{\|, f}\right\rangle$ can be determined as outlined above. Measurements at different values of $B_{f}$ yield the final longitudinal energy as a function of $\alpha$. The initial mean perpendicular energy $\left\langle E_{\perp, i}\right\rangle$, being the quantity of interest, can then be obtained via the relation

$$
\left\langle E_{\perp, i}\right\rangle=-\frac{\mathrm{d}\left\langle E_{\|, f}\right\rangle}{\mathrm{d} \alpha} .
$$

In the above discussion, we have implicitly assumed that the initial $E_{\|}$and $E_{\perp}$ are characterized by independent distribution functions. The quoted results, in particular Eq. (6), remain valid also if correlation exists between the two quantities. The distribution function for $E_{\|}$under adiabatic transport into another magnetic field will behave very differently, though. Some of our results suggest such correlation, as will be detailed later.

\section{Experimental set-up}

For our experiments we used two beams with quite different characteristics from the NEPOMUC facility: the high-energy, high-intensity primary beam from a Pt moderator upstream in the source and the low-energy, high-brightness remoderated beam which can optionally be produced by remoderation from a W crystal in reflection geometry [12]. Geometrically this remoderator set-up is located between the primary Pt source and the position of the user experiments. The kinetic energy of the positrons within NEPOMUC is set, for the primary beam, as the potential difference between the extracting Pt structure and the beam line [9]. For the remoderated beam, the energy is set by biasing the $\mathrm{W}$ remoderator crystal accordingly. Nominal energy values quoted for the remoderated beam include the negative positron work function of $\mathrm{W}$ of about $-2.9 \mathrm{eV}$ $[12]$.

Either of the two beams is transported in a magnetic guiding field, which in our case was $5 \mathrm{mT}$ on axis, to the experimental set-up. Our experimental set-up was installed at the open beam port, a port available for external user experiments. The set-up is sketched in Fig. 1. During experiments, an operating pressure of $10^{-7}$ mbar was achieved using two turbomolecular pumps. In the following section, the main components of the experimental set-up are described in more detail.

\subsection{Magnetic guiding field}

An arrangement of ten magnetic field coils was designed to meet the following requirements: match the 5-mT guiding field of the upstream NEPOMUC beam line, maintain homogeneity at the location where the beam profile and intensity are determined, provide a section with an adjustable adiabatic field gradient and provide a final uniform field section for the energy measurements. Examples of two magnetic field configurations used in the measurements are shown in Fig. 2. In the experiments the coil currents were chosen to closely match the 5 -mT 


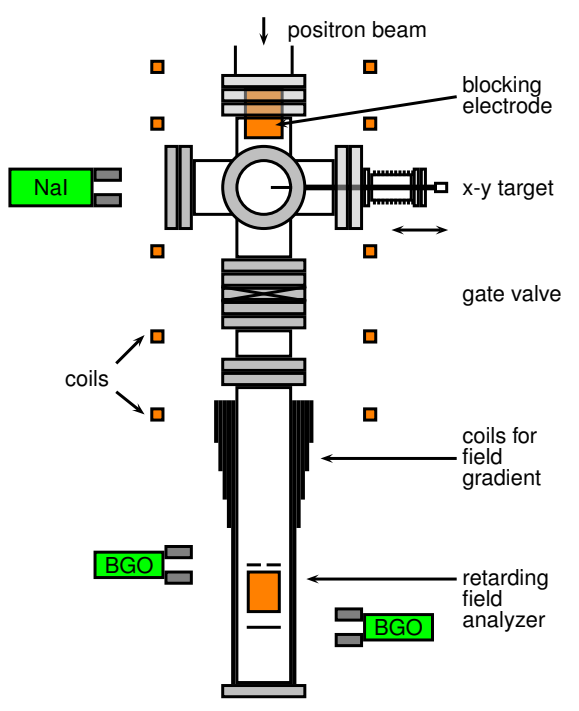

Fig. 1: Experimental set-up installed at the open beam port of the NEPOMUC beam line (not to scale). Main components are the coil system for magnetic guiding, the $\mathrm{x}-\mathrm{y}$ targets for beam intensity and profile measurements and the retarding field analyzer for energy measurements.

guiding field which resulted in calculated on axis values of $5.1 \mathrm{mT}$ at the target position and $4.8 \mathrm{mT}$ at the RFA position. Some ripple in the magnetic field lines due to imperfections of our coil arrangement is seen in the Fig. 2, with the worst being at $z \approx 0 \mathrm{~m}$. In experiments with the primary beam, an additional coil was used for mitigation of the ripple in this region. We have carried out extensive numerical calculations to quantify how strongly this affects our results and will comment on this point below.

\subsection{Target chamber}

To determine the intensity and spatial extent of the beam a six-way cross was installed, housing a cylindrical copper electrode for beam blocking at the entrance and two rectangular stainless steel targets that can be moved in two orthogonal directions in the plane perpendicular to z, as illustrated in Fig. 3.

In order to determine its intensity, the entire beam was collected for a fixed amount of time on one of the fully inserted targets. For diagnosis, either the current collected on the plate was measured with a charge-integrating amplifier (operational amplifier AD820 with 4.7-nF feedback capacitor) or the 511-keV annihilation-radiation signal was recorded by a 2" x2" NaI scintillation detector from Ortec with a digiBASE system. For the beam profile measurement, the method relying on the annihilation signal was typically used. The targets A and B, see Fig. 3, were translated through the beam and the corresponding counts 

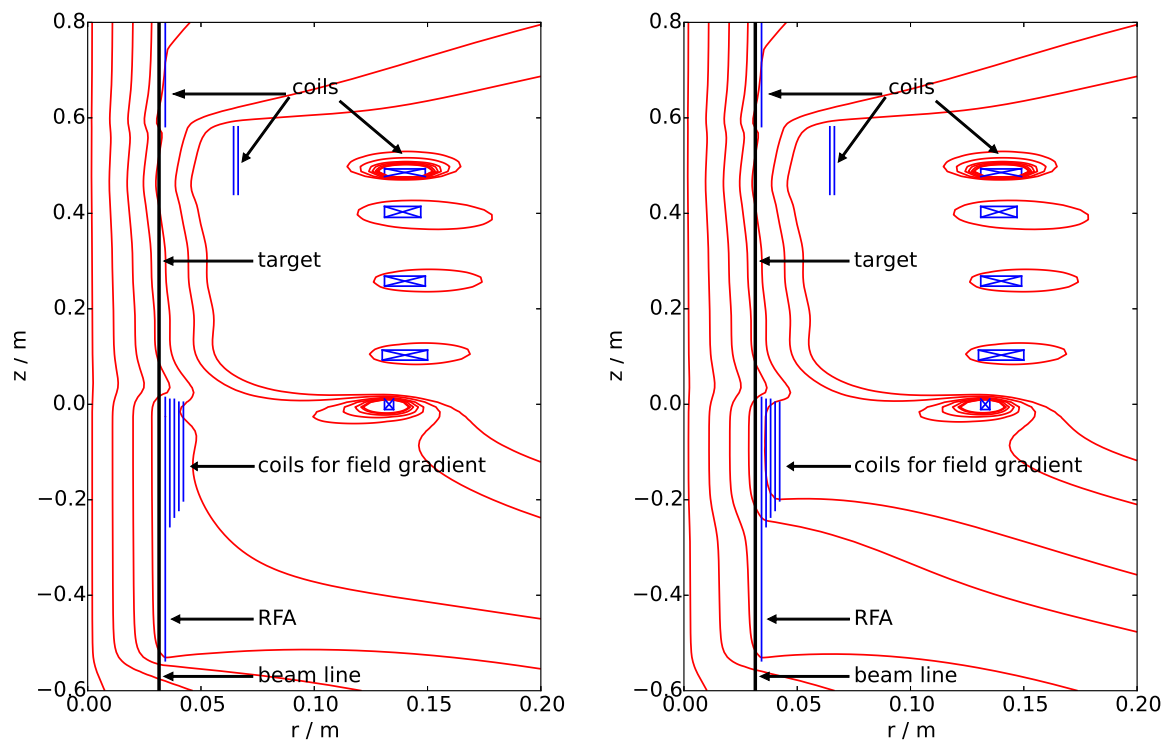

Fig. 2: Cross-section through the set of magnetic field coils (blue) and representative magnetic field lines (red). The set-up has cylindrical symmetry with the rotation axis at $r=0 \mathrm{~m}$. The vertical black line indicates the beam line boundary. The positron beam enters from the top. The beam intensity and spread are measured at $z \approx 0.3 \mathrm{~m}$ (label "target") where the magnetic field on axis is $5.1 \mathrm{mT}$. The energy measurement is done in the section with $-0.6 \mathrm{~m} \leq z \leq 0.0 \mathrm{~m}$. Here the coils for the field gradient are capable of creating both a homogeneous field of $4.8 \mathrm{mT}$ on axis (left) and various magnetic field gradients (right). The RFA is located at $z \approx-0.45 \mathrm{~m}$.

in the annihilation peak were recorded.

\subsection{Retarding field analyzer}

The energy measurements were performed with a retarding field analyzer (RFA), see Fig. 1, consisting of a stainless steel aperture; a cylindrical, goldplated retarding electrode with a grid of $65 \%$ transmittance on the downstream side; and a stainless steel collector that could be biased to assure collection of low-energy positrons. The aperture was initially implemented into the set-up to possibly yield a better energy resolution. As the alignment especially of the primary beam with this aperture proved difficult, various aperture diameters were tested. The measurements here include diameters of 5,15 and $30 \mathrm{~mm}$. The annihilation signal produced on the collector for a range of voltages applied to the retarding electrode was detected through a $4 \mathrm{~mm}$ wide collimator with a BGO scintillation detector $(d=22 \mathrm{~mm}, l=25 \mathrm{~mm}$, from Korth Kristalle, with Hamamatsu type H10425 photomultiplier). Signal processing was done using an Ortec type 855 amplifier and a type 551 timing SCA that fed a digital counter. All measurements were taken with a grounded aperture. The collector was 


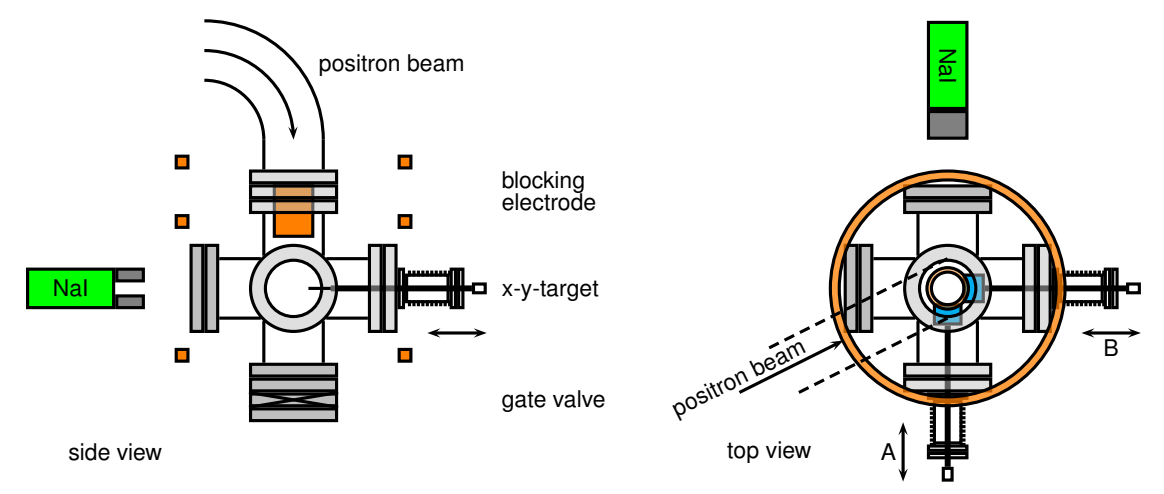

Fig. 3: Side (left) and top view (right) of the six-way cross housing an electrode for beam blocking at the beam entrance and two insertable targets on perpendicular axes in the midplane. The target movement is realized by mechanical feedthroughs. Either the current collected on the target or the accompanying annihilation radiation can be detected by a charge integrating amplifier or a NaI scintillation detector, respectively.

biased either to $0 \mathrm{~V}$ or to a negative voltage to ensure a minimum implantation energy. For measurements with the primary beam the collector was biased to $0 \mathrm{~V}$.

\section{Numerical calculations}

As the magnetic guiding field in our set-up is created by several individual coils, giving rise to inhomogeneous field sections in the connection regions, it is not a priori clear whether adiabatic guiding of the positrons can be assumed. The adiabaticity of the guiding field is preserved if the magnetic field experienced by the parallel-streaming particles does not vary significantly over one gyration length $\lambda_{\|}$. According to [14] an adiabaticity parameter can be constructed which is a quantitative measure of this property:

$$
\xi=\frac{\lambda_{\|}}{B}\left|\frac{\mathrm{d} B}{\mathrm{~d} z}\right| \ll 1,
$$

with $\lambda_{\|}=2 \pi m v_{\|} / q B, B$ the magnetic field at the particle position, $\mathrm{d} B / \mathrm{d} z$ the gradient of the magnetic field along the field direction, $v_{\|}$the parallel velocity of the particle and $m$ and $q$ the particle mass and charge. From Eq. (7) it is clear that less stringent requirements on the field homogeneity can be imposed for particles with low kinetic energies. Inspection of $\xi$ in our set-up suggests nonadiabatic guiding for energies typically applied for the primary beam, mainly 
caused by the ripple in the guiding field at $z=0 \mathrm{~m}$. For a kinetic energy of $400 \mathrm{eV}$ we found for example a maximal value of $\xi \sim 1.1$ at $z=0 \mathrm{~m}$. The installation of an additional coil (not shown in Fig. 2) seemingly improved adiabaticity for these energies. For a kinetic energy of $400 \mathrm{eV}$ we now find $\xi \sim 0.1$ at $z=0 \mathrm{~m}$ and $\xi \lesssim 0.23$ in our entire set-up. In order to verify adiabatic guiding in our set-up, we have carried out numerical orbit calculations detailed in the following section.

\subsection{Initial particle distribution}

For the initial particle distribution 2000 particles were generated randomly with a Gaussian spatial profile centered on the magnetic axis. The spatial extent was comparable to the beam diameter we determined for the primary beam, i.e. $\sigma=5 \mathrm{~mm}$. A Gaussian distribution was assumed for the longitudinal energy

$$
f\left(E_{\|}\right)=\frac{1}{\sqrt{2 \pi} \sigma_{\|}} \exp \left(-\frac{\left(E_{\|}-\left\langle E_{\|}\right\rangle\right)^{2}}{2 \sigma_{\|}^{2}}\right)
$$

with $\left\langle E_{\|}\right\rangle$and $\sigma_{\|}$the mean longitudinal energy and spread, respectively. The perpendicular energy distribution was described by a Maxwell-Boltzmann distribution

$$
f\left(E_{\perp}\right)=\frac{1}{k_{b} T_{\perp}} \exp \left(-\frac{E_{\perp}}{k_{b} T_{\perp}}\right)
$$

with $T_{\perp}$ the perpendicular temperature and $k_{b}$ Boltzmann's constant. The spread $\sigma_{\|}$of the longitudinal distribution was set equal to $\left\langle E_{\perp}\right\rangle=k_{b} T_{\perp}$ in the perpendicular distribution.

\subsection{Magnetic field and equations of motion}

The magnetic field was calculated analytically using the Biot-Savart law for current loops with the coil parameters used in our experiments. At each time step, after calculating the magnetic field, the positions and velocities of the particles were updated using an integrator scheme based on the Boris algorithm $[15]$.

\subsection{Calculation results}

In order to determine for which energies our set-up yields adiabatic guiding, when no field gradient is applied for $z<0$, we calculated the magnetic moment, $\mu=E_{\perp} / B$, of each particle after each time step and averaged it over an initial homogeneous field section. This was compared to the magnetic moment averaged over the final homogeneous field section. The result is shown in Fig. 4 for different total energies with the perpendicular temperature fixed to $1 \mathrm{eV}$ and a spatial standard deviation of $5 \mathrm{~mm}$.

For adiabatic guiding, the initial and final magnetic moments should be equal and all points should fall on a line with unit slope. Particles with energies greater than about $100 \mathrm{eV}$ are not transported adiabatically and thus show deviations from the line with slope unity. To investigate if this is caused by the 

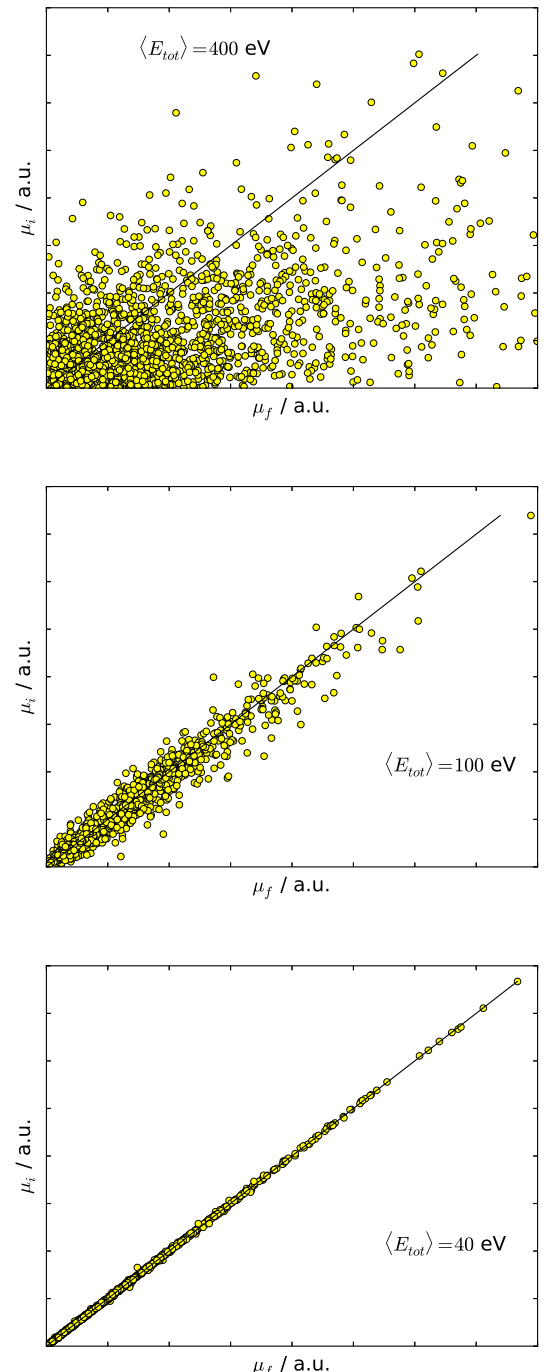

Fig. 4: Numerically calculated magnetic moments for 2000 particles in the initial versus the final section of our set-up. Three different mean total energies are shown. The mean transverse energy was fixed to $1 \mathrm{eV}$ and the spatial standard deviation was $5 \mathrm{~mm}$. The conservation of the magnetic moment is given by the solid line.

off-axis inhomogeneities and thus the spatial spread of the particles, the spatial standard deviation was varied between 1 and $5 \mathrm{~mm}$ at fixed mean total and perpendicular energy. We found that the deviations of the final from the initial magnetic moment decreased with decreased spatial spread. However, the effect 
was not sufficient to explain the non-adiabatic transport.

Another important parameter seems to be the ratio of the mean transverse energy (i.e. perpendicular temperature), and thus the longitudinal spread, to the mean longitudinal energy. For high total beam energies and very small transverse temperatures, the parallel energy distribution develops a low-energy shoulder when it passes through our set-up. For the same total energy with a higher perpendicular temperature, and thus higher longitudinal spread but lower mean longitudinal energy, there is still some distortion of the parallel energy distribution but the general shape is conserved, see Fig. 5.
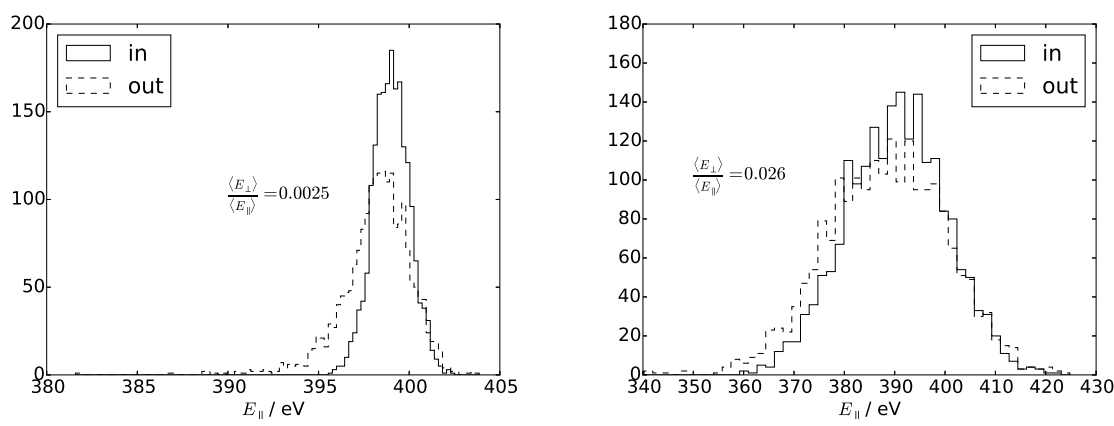

Fig. 5: Numerical calculation of the parallel energy distribution for 2000 particles with spatial standard deviation of $5 \mathrm{~mm}$ before (solid line) and after (dashed line) transport through our set-up. The mean total energy was fixed to $400 \mathrm{eV}$. The longitudinal spread and mean perpendicular energy were set equal. Two different values of the longitudinal spread are shown (left: $1 \mathrm{eV}$, right: $10 \mathrm{eV}$ ), leading to two different ratios for the mean perpendicular to the mean parallel energy.

In order to conduct energy measurements for high-energy beams we must determine to what degree we can tolerate lack of conservation of the magnetic moment. We therefore simulated the measurement of the longitudinal energy distribution by varying $V_{R}$ and counting only particles with $E_{\|}>V_{R}$. Using the same methods we used for the analysis of the experimental data, we determined the mean longitudinal and transverse energy, as well as the energy spread, and compared them to the initial values, see Fig. 6. For all tested energies, the "measurement" of the mean longitudinal energy seems to be very robust even against distortions of the energy distribution. This is indicated by the line of slope unity in the upper panel of Fig. 6.

For total energies of the order of $100 \mathrm{eV}$ and below, the transverse energy and the energy spread deviate by about $10 \%$ from the initial parameters. For higher beam energies, care must be taken when interpreting the results, in particular for beams with very low transverse temperatures.

In summary, the simulations show that the non-adiabatic transport in the analyzing device results in about $10 \%$ changes in the longitudinal spread and mean transverse energy for positron energies not exceeding $\sim 100 \mathrm{eV}$. The determination of the mean longitudinal energy seems not to be influenced by the non-adiabatic transport. 

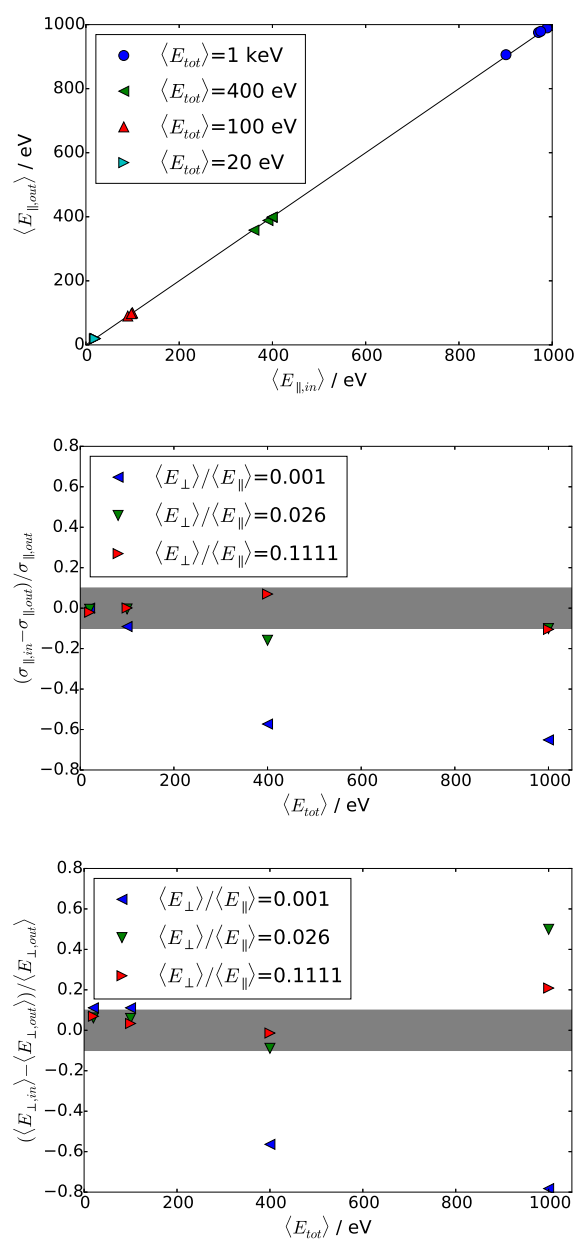

Fig. 6: Comparison of the initial energy parameters of the numerical calculation with the parameters determined from a simulation of the measurement process in our RFA. The spatial spread was fixed to $5 \mathrm{~mm}$. (Top) Comparison of the "measured" mean longitudinal energy to the initial one. Points of the same color show the variation for different mean transverse energies and energy spreads at fixed mean total energy. The line is of slope unity. (Middle) Relative deviation of the mean energy spread for various mean total energies and three different ratios of the mean perpendicular energy to the mean longitudinal energy. The gray band corresponds to a deviation of less than $10 \%$. (Bottom) Relative deviation of the mean transverse energy for various mean total energies and three different ratios of the mean perpendicular energy to the mean longitudinal energy. The gray band corresponds to a deviation of less than $10 \%$.

\section{Experimental results}

\subsection{Beam intensity}

The beam intensity was determined with both the charge-integrating amplifier, hereafter referred to as method 1 , and the scintillation detector, referred to 
as method 2. For method 1 the output of the charge-integrating amplifier was digitized with an oscilloscope (Tektronix MDO3024); a typical signal is shown in Fig. 7.

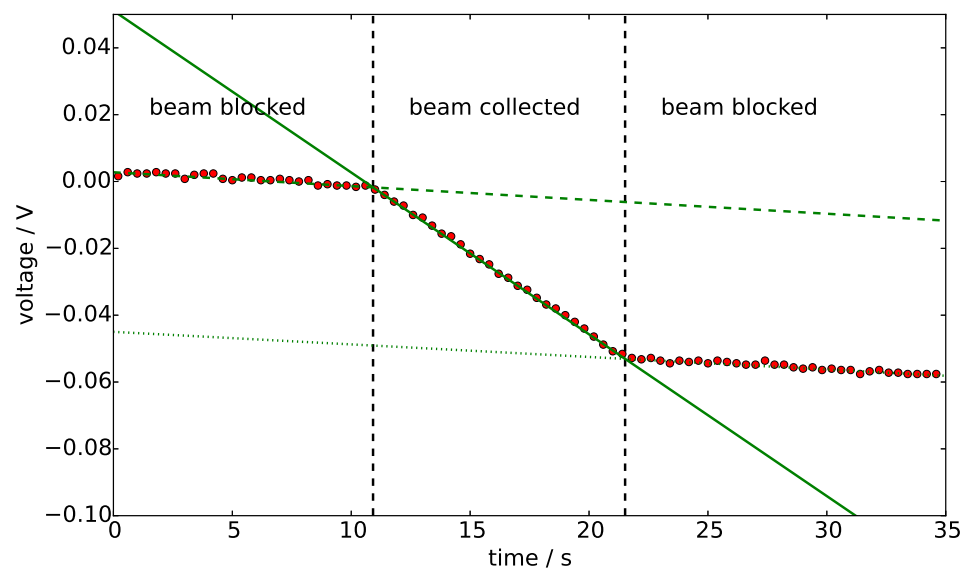

Fig. 7: Typical charge-integrating amplifier output measured on an oscilloscope. Initially the beam is blocked by biasing a cylindrical electrode to a potential higher than the beam energy, then collected by grounding the same electrode and finally blocked again. For data analysis only every 20 th point was used and the three regions were fitted with linear functions. Here $1 \mathrm{mV} / \mathrm{s}$ corresponds to $3.1 \cdot 10^{7} e^{+/ \mathrm{s}}$

Initially, by applying a positive bias higher than the beam energy to the entrance electrode, the beam was blocked and only the intrinsic drift of the charge-integrating amplifier was observed. By collecting the beam on the target the slope of the signal changes according to the rate of collected charge. As soon as the beam is blocked again, the signal recovers the intrinsic drift. After correction for the drift, the beam intensity is obtained from the characteristics of the integrating circuit, with $1 \mathrm{mV} / \mathrm{s}$ corresponding to $(3.1 \pm 0.1) \cdot 10^{7} e^{+} / \mathrm{s}$. Here, we do not take into account that a part of the positron beam is backscattered from the target and thus does not contribute to the signal. Typically, this fraction lies in the order of $10 \%$ for a $1-\mathrm{keV}$ positron beam and seems to decrease with decreasing beam energy [16].

For method 2, the beam was collected for $30 \mathrm{~s}$ and the count rate in the $511 \mathrm{keV}$ annihilation peak was recorded. These data were converted to absolute units by comparison to a ${ }^{22} \mathrm{Na}$ calibration source in the same geometry. The activity of the ${ }^{22} \mathrm{Na}$ source, which yields positrons in $90 \%$ of the decays, was $2.3 \cdot 10^{5} \mathrm{~Bq}$ and an uncertainty of $10 \%$ was assumed. Again, the backscattered positrons cannot be detected. In addition, with this method we miss those gamma rays originating from the $3 \gamma$ decay of ortho-Positronium, as well as those piling up in the detector. Combining all three effects leads to a maximum correction factor of about 2 [11]. 


\begin{tabular}{|c|c|c|}
\hline \hline energy / eV & method 1/ $e^{+} / \mathrm{s}$ & method $2 / e^{+} / \mathrm{s}$ \\
\hline $5^{a}$ & $2.3(2) \cdot 10^{7}$ & $2.8(5) \cdot 10^{7} b$ \\
12 & - & $2.8(5) \cdot 10^{7}$ \\
22 & $6.5(6) \cdot 10^{7}$ & $3.2(6) \cdot 10^{7}$ \\
400 & $5.4(1) \cdot 10^{8}$ & - \\
$1000^{c}$ & $1.2(1) \cdot 10^{8}$ & $9(2) \cdot 10^{7}$ \\
$1000^{d}$ & $3.0(1) \cdot 10^{8}$ & - \\
\hline \hline
\end{tabular}

Table 1: Beam intensities of the NEPOMUC beam at different energies determined by two methods: a charge integrating amplifier (method 1) and a NaI scintillation detector (method 2). Corrections due to backscattering, $3 \gamma$ or pile-up events are not included. For further details, see Section 5.1. ${ }^{a}$ Different magnetic configurations below the target for method 1 and 2. ${ }^{b}$ Target biased to $-7 \mathrm{~V} .{ }^{c}$ Beam time $1 .{ }^{d}$ Beam time 2 .

Hence, our results, which are summarized for both methods and the various beam energies in Table 1 without the aforementioned corrections, represent a lower limit of the true beam intensity at the open beam port. In general, both methods yield comparable orders of magnitude for the beam intensity. It should be noted that the remoderated beam with energies up to $22 \mathrm{eV}$ naturally has a reduced intensity of about $3 \cdot 10^{7} e^{+} / \mathrm{s}[11]$ due to the limited remoderation efficiency. For the 5-eV beam, against expectations, the intensity observed with method 2 is higher than with method 1 . This might be attributed to the negative target bias, only applied while using method 2, which leads to an improved focus particular for low-energy positrons and the re-attraction of backscattered positrons. In addition, the magnetic configuration installed below the target was different while using method 1 and 2 . The maximum intensity for the remoderated beam at our set-up was observed with both methods for a beam energy of $22 \mathrm{eV}$. This energy is the one used for most other experiments at NEPOMUC and thus was already subject to many optimization steps.

The highest positron flux in our set-up was measured for the primary beam at $400 \mathrm{eV}$. Our figure is significantly smaller than estimates from a Monte-Carlo simulation of the current source geometry $\left(3 \cdot 10^{9} e^{+} / \mathrm{s},[10]\right)$ and reduced in comparison to an experimental value measured earlier with a beam energy of $540 \mathrm{eV}\left(1.1 \cdot 10^{9} e^{+} / \mathrm{s},[11]\right)$. These values, however, refer to the intensity at positions several meters upstream of the current location of user experiments. We therefore attribute the loss of positrons to the non-adiabatic guiding of the high-energy beam through several narrow bends to the present open beam port. We understand this also to be the reason that the intensity of the $1 \mathrm{keV}$ beam is at most $55 \%$ of the intensity of the $400-\mathrm{eV}$ beam. Furthermore, the difference in the results from two different beam times indicate that the beam intensity of the $1-\mathrm{keV}$ beam is sensitive to the beam optimization.

In summary, we observed a similar and even higher intensity for the remoderated beam with energies up to $22 \mathrm{eV}$ than the one previously measured close to the source [11]. The beam guiding of this low-energy beam to the user experiments thus seems to be already very efficient. In contrast, the primary beam shows 
energy-dependent losses which suggest a non-adiabatic transport from the source to the user experiment. To fully benefit from the high intensity of this beam, further optimization, i.e. lowering of the source potential and hence the kinetic energy of the positrons, is required.

\subsection{Beam profile}

Beam profile measurements were primarily conducted using method 2 by repeating the measurement described in section 5.1 for each target position. The intensity of the $1-\mathrm{keV}$ beam allowed in addition a measurement with method 1 by step-wise repeating the measurement described in the previous section. During the present measurements only one-dimensional cuts of the beam profile were recorded, such as those shown in Fig. 8 and Fig. 9.
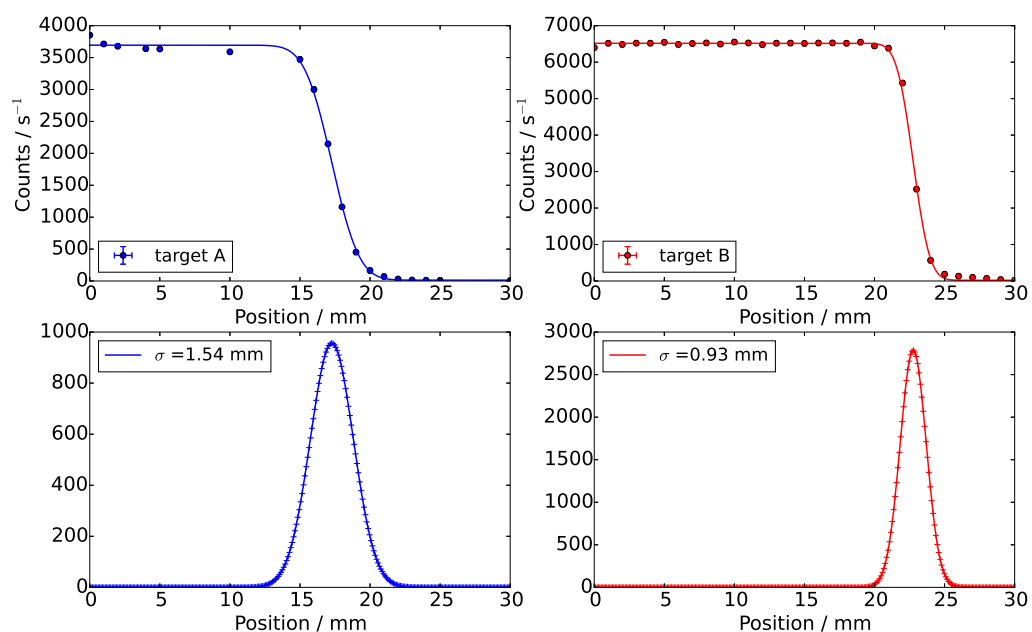

Fig. 8: Typical one-dimensional beam profiles obtained by translating a target in either 'A' or ' $\mathrm{B}$ ' direction, see Fig. 3, through the beam and detection of the annihilation radiation. This example shows the remoderated beam with an energy of $5 \mathrm{eV}$. In the upper row, the data was fitted assuming a Gaussian profile, as in Eq. (1). The lower row shows the derivative of the fit. The center of the chamber is at $25 \mathrm{~mm}$.

The full width at half maximum (FWHM), obtained from the Gaussian width by $\mathrm{FWHM}=2 \sqrt{2 \ln 2} \sigma$, is summarized for all beam energies in the $5.1-\mathrm{mT}$ guiding field, which was present on axis at the target position, in Table 2. The remoderated beam with energies up to $22 \mathrm{eV}$ is found to have a very narrow beam profile, with a FWHM less than $3.8 \mathrm{~mm}$, that is nearly independent of the beam energy. In contrast, the primary beam at $1 \mathrm{keV}$ has a broader distribution, with a FWHM in the order of $14 \mathrm{~mm}$, as confirmed with both methods. The broad profile further complicates the efficient guiding of the beam.

Although the one-dimensional measurements reveal the position and the general elliptical shape of the beam, the rotation of the ellipse in the measurement plane 

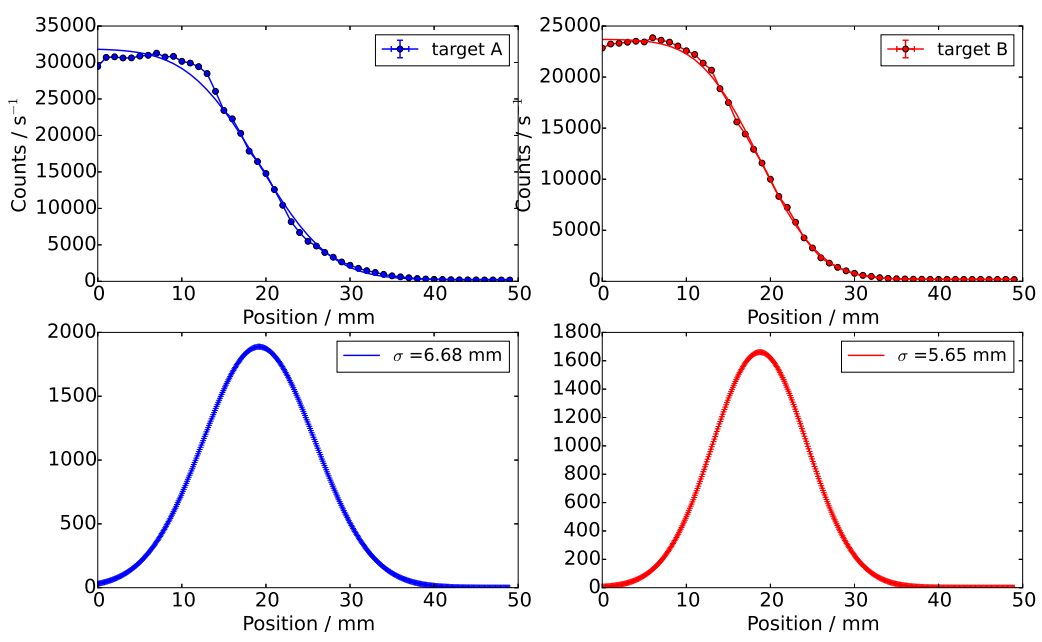

Fig. 9: Similar beam profiles as in Fig. 8 for the primary beam with an energy of $1 \mathrm{keV}$.

remains unresolved. Thus, the beam shape could be any ellipse that fits into the rectangle in the measurement plane defined by the perpendicular FWHM values from our measurements. Measurements addressing the $2 \mathrm{D}$ beam shape are planned for the future.

\begin{tabular}{|c|cc|c|}
\hline \hline energy / eV & FWHM $_{A} / \mathrm{mm}$ & FWHM $_{B} / \mathrm{mm}$ & method \\
\hline 5 & $3.6(1)$ & $2.2(1)$ & 2 \\
12 & $3.0(1)$ & $2.1(1)$ & 2 \\
22 & $3.54(8)$ & $2.60(5)$ & 2 \\
1000 & $15.7(4)$ & $13.3(1)$ & 2 \\
1000 & - & $14(1)$ & 1 \\
\hline \hline
\end{tabular}

Table 2: Beam width determined for the NEPOMUC beam in a $5.1 \mathrm{mT}$ magnetic guiding field at various beam energies. Two perpendicular targets were translated through the beam and the corresponding positron signal was recorded by a charge integrating amplifier (method 1) or a $\mathrm{NaI}$ scintillation detector (method 2). Only statistical uncertainties are given. Indices $A$ and $B$ refer to scanning directions as shown on the right in Fig. 3.

\subsection{Energy distribution}

After moving the beam to the center of the target chamber (with an offset of $2 \mathrm{~mm}$ at most), the energy distribution was determined with the retarding field analyzer. For each beam energy, the annihilation signal of positrons hitting the collector was recorded for a range of values of the retarding potential. Measurements for the beam at 5 and $12 \mathrm{eV}$ were taken with a 5 -mm diameter aperture 
and four different values of the final magnetic field $B_{f}$. For measurements of the $22-\mathrm{eV}$ beam, the aperture was $30 \mathrm{~mm}$ in diameter and five different values of $B_{f}$ were used. For the $400-\mathrm{eV}$ beam, a $15-\mathrm{mm}$ diameter aperture was used, and three different $B_{f}$ were applied.

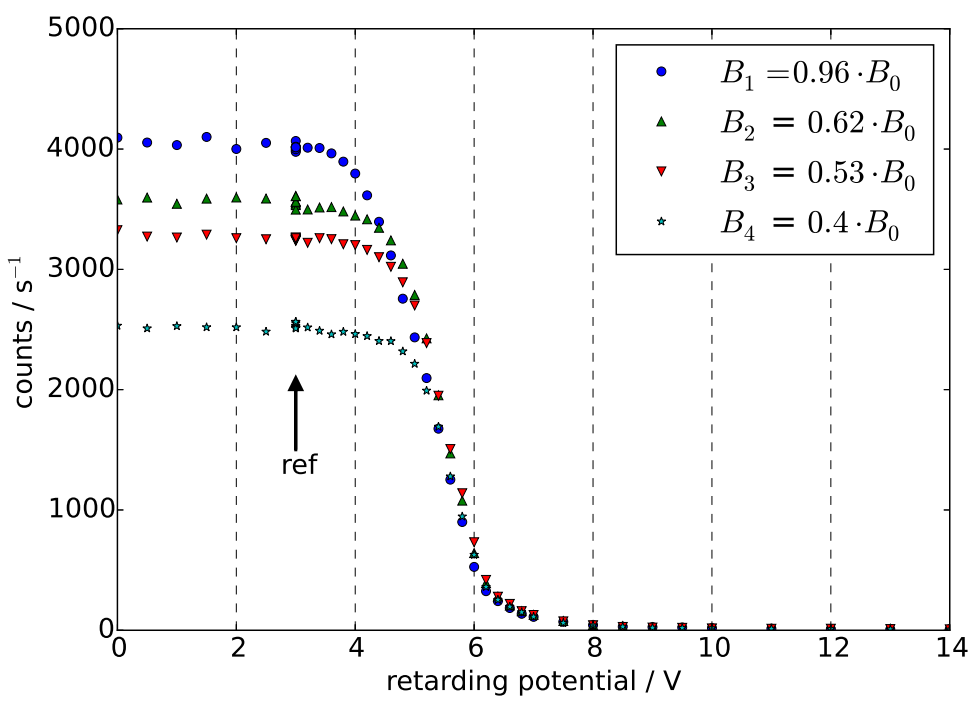

Fig. 10: Detected beam intensity as function of retarding field potential for the $5 \mathrm{eV}$ beam in four different magnetic field configurations with $B_{1}>B_{2}>B_{3}>B_{4}$ at the measurement position and $B_{0}=5 \mathrm{mT}$. The arrow indicates the voltage at which reference measurements were taken periodically. In these measurements the diameter of the aperture was $5 \mathrm{~mm}$ and the collector was biased to $-20 \mathrm{~V}$.

Typical signals from the retarding field analyzer are shown for the 5 -eV beam in the different final magnetic fields in Fig. 10. Here, the collector was biased negatively. The reduced signal for smaller final magnetic field might result from two different effects: (I) an increase of the beam diameter when it is guided into a lower field region, as

$$
B \cdot r^{2}=\text { const. }
$$

and (II) a remaining misalignment of the beam and the RFA. Both of these effects would increase particle losses at the aperture when the final magnetic field is smaller. The beam intensity was monitored during the measurements by repeating measurements at a reference value for the retarding field potential, as indicated by the arrow in Fig. 10, and, within one set of conditions, was found to be stable over time.

With decreasing magnetic field, the slope of the signal vs. retarding voltage becomes steeper, while the retarding voltage which is necessary to block the entire beam does not change. This behavior could not be explained if the parallel and perpendicular energy distributions were fully independent. In other words, 
our data show that parallel and perpendicular energy are correlated, and they add up to a total energy distribution, which is narrower than the two individual distributions.

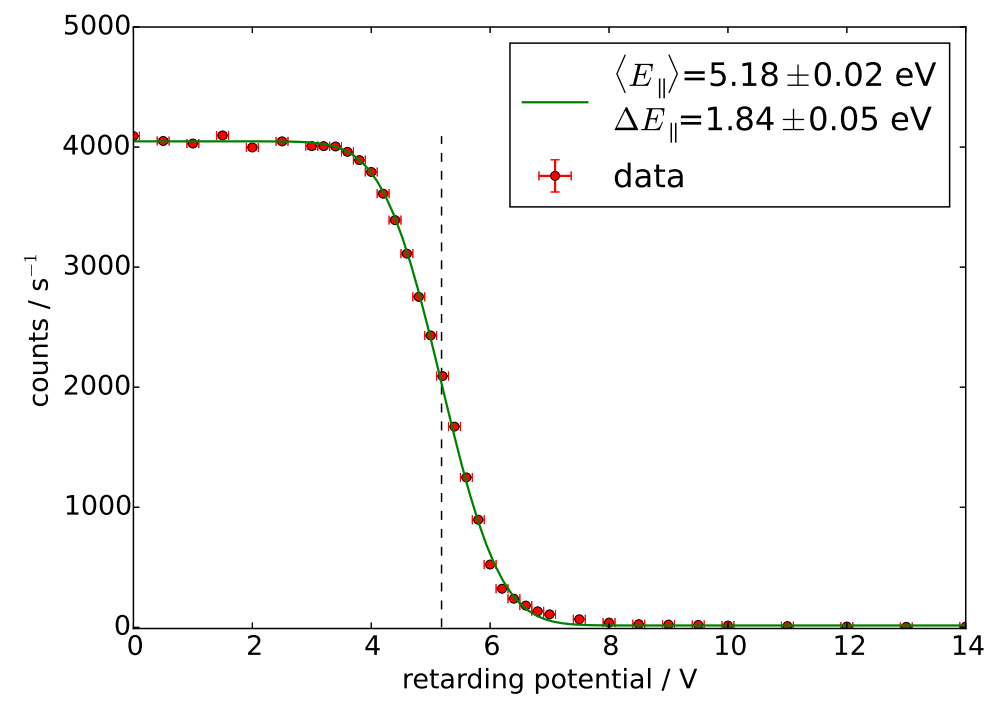

Fig. 11: Detected beam intensity as function of retarding field potential for the 5-eV beam in the homogeneous guiding field of $4.8 \mathrm{mT}$ with an aperture of $5 \mathrm{~mm}$ and the collector biased to $-20 \mathrm{~V}$. The data is fitted by Eq. (3) yielding a mean longitudinal energy of $5.18(2) \mathrm{eV}$.

The data from the retarding field analyzer were fitted assuming a Gaussian energy distribution function, see Eq. (3), to yield the mean parallel energy and the corresponding energy spread, as shown in Fig. 11. From the variation of the mean parallel energies with the field ratio $\alpha$, see Fig. 12, the mean perpendicular energy can be extracted using Eqs. (5) and (6). The intersection of the fit function with the $y$ axis corresponds to the case where the perpendicular energy is completely converted to parallel energy and thus is a measure for the mean total beam energy $\left\langle E_{t o t}\right\rangle$.

As the magnetic field at the RFA is slightly lower than the one in the NEPOMUC beam line, the mean longitudinal energy in the 5-mT guiding field, corresponding to $\alpha=1$, has to be extracted from Eq. (5) as well. The results for the remoderated beam at the three different energies are summarized in Table 3. For completeness we include also the beam brightness which can be defined [9] as

$$
B^{*}=\frac{I}{d^{2} E_{\perp}}
$$

with $I$ the beam intensity and $d$ the beam diameter. For each energy, we used the intensity obtained with method 2, the mean spread from Table 2 normalized 


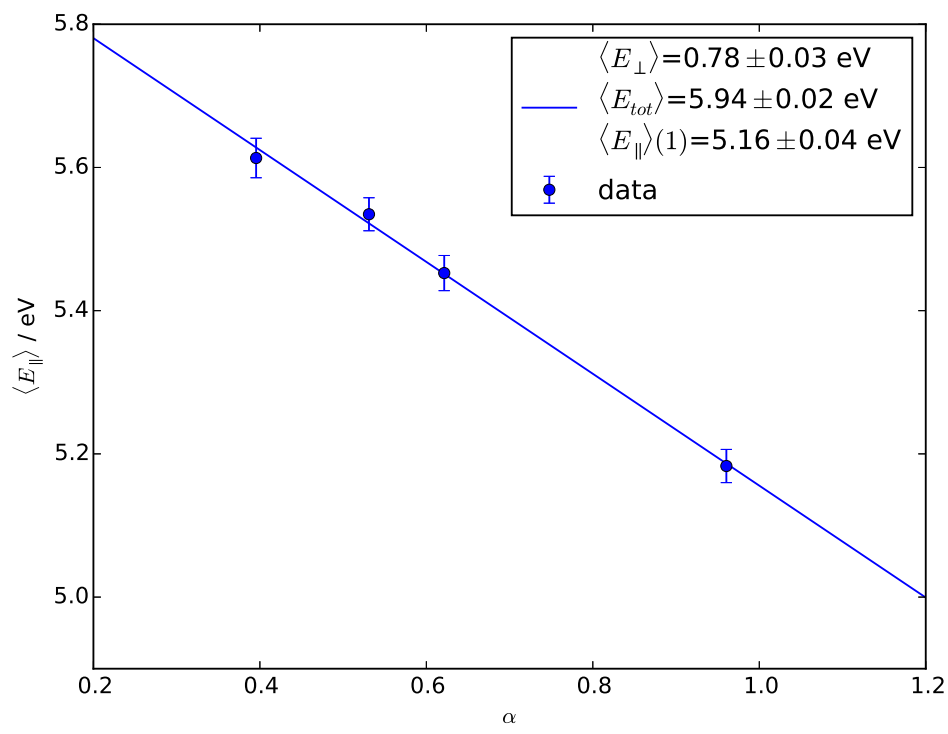

Fig. 12: Variation of the mean parallel energy with different magnetic field ratios $\alpha=B_{f} / B_{i}$ and $B_{i}=5 \mathrm{mT}$ for the $5 \mathrm{eV}$ beam with an aperture of $5 \mathrm{~mm}$ and a negatively biased collector. From a linear fit according to Eq. (5) the initial mean perpendicular, total and longitudinal energy can be determined.

to the 5-mT field by Eq. (10) and the mean perpendicular energy to arrive at the values in Table 3 .

\begin{tabular}{|c|c|c|c|c|c|}
\hline \hline energy / eV & $\left\langle E_{\|, i}\right\rangle / \mathrm{eV}$ & $\Delta E_{\|} / \mathrm{eV}$ & $\left\langle E_{\perp, i}\right\rangle / \mathrm{eV}$ & $\left\langle E_{\text {tot }}\right\rangle / \mathrm{eV}$ & $B^{*} / \mathrm{mm}^{-2}(\mathrm{eVs})^{-1}$ \\
\hline 5 & $5.16(4)$ & $1.8(1)$ & $0.78(3)$ & $5.94(2)$ & $4.2(8) \cdot 10^{6}$ \\
12 & $11.77(7)$ & $2.5(2)$ & $1.22(6)$ & $12.98(4)$ & $3.5(7) \cdot 10^{6}$ \\
22 & $20.7(1)$ & $3.1(2)$ & $1.3(1)$ & $22.05(7)$ & $2.6(5) \cdot 10^{6}$ \\
\hline \hline
\end{tabular}

Table 3: Parameters for the energy distribution of the NEPOMUC remoderated beam at different energies in a $5 \mathrm{mT}$ magnetic guiding field as it arrives at the open beam port. In addition the beam brightness $B^{*}$ is given, for definition see Eq. (11). $\left\langle E_{\|}\right\rangle,\left\langle E_{\perp}\right\rangle$ and $\left\langle E_{\text {tot }}\right\rangle$ are determined from the fit with Eq. (5) as shown in Fig. 12. $\Delta E_{\|}$is determined from the fit of Eq. (3) as shown in Fig. 11. Only statistical uncertainties are given except for $\Delta E_{\|}$where a systematic uncertainty of $4 \%$ is included because of the difference in $B$ field.

Surprisingly, for the remoderated beam at 5 and $12 \mathrm{eV}$ there was an offset of about 0.9 to $1 \mathrm{eV}$ in the mean total energy, compared to the energy set at the remoderation crystal, which is the difference of the acceleration potential and the positron work function. Obvious sources for such an offset were ruled out. We note that differences in the positron work function between the $\mathrm{W}$ 
remoderator and the Au RFA electrode can play a role in this measurement, as well as contact potential differences between the positron beam facing materials. The latter will be determined by differences in the electron work functions. We further recall that the $22-\mathrm{eV}$ data were taken with a large $(\mathrm{d}=30 \mathrm{~mm})$ stainless steel aperture in front of the RFA, while beams with 5 and $12 \mathrm{eV}$ used a small aperture $(\mathrm{d}=5 \mathrm{~mm})$. The lower energy beams are therefore more likely to be influenced by the contact potential difference between stainless steel and gold, which has a value of $0.8 \mathrm{eV}$ and is accelerating positrons towards the $\mathrm{Au}$ electrode (electron work functions of stainless steel $4.3 \mathrm{eV}$ [17], polycristalline $\mathrm{Au} 5.1 \mathrm{eV}[18])$. Although this may explain the appearance of a difference between the data sets, in view of the many conceivable effects that could lead to energy changes of a similar magnitude, e.g. oxide coverage of electrodes, influence of the positron work function for $\mathrm{Au}(+1.1 \mathrm{eV},[19])$ or fringing field effects, we have to call this a speculative explanation, to be explored in future experiments.

The observed energy spread for the remoderated beam and its increase with increasing beam energy is unexpectedly large, given that the gradient of the surface potential of the $\mathrm{W}$ remoderator crystal is steep, leading to positron emission mainly in the direction of the surface normal. Thus, a perpendicular energy spread on the order of the remoderator temperature is expected. This however only holds when the the crystal is perfectly flat, the extracting electric field and the magnetic guiding field are exactly perpendicular to the $\mathrm{W}$ crystal surface and if the subsequent beam guiding does not affect the energy distribution. During its general use, the remoderation crystal undergoes several cycles of conditioning which in the course of time might lead to deformation of the surface. Positrons emitted from such surface would then naturally acquire a broader energy spread. In addition, the beam guiding from the remoderator crystal back into the beam line involves fields that are inhomogeneous and bent [12]. Together with the aforementioned surface-geometry effects this might well account for the observed energy spread seen at the open beam port.

For the primary beam at a nominal energy of $400 \mathrm{eV}$, we show a set of measured data in Fig. 13. The reduction in beam intensity at the RFA detector is seen for retarding voltages $V_{R}$ as low as $300 \mathrm{~V}$. This suggests that the beam has a low-energy tail. In the three different magnetic fields we clearly observe a conversion of perpendicular to parallel energy. However, due to the asymmetry, an analysis of the data with the methods outlined above for the remoderated beam, in particular fitting the data with the distribution function of Eq. (3), did not produce conclusive results. In our data analysis, we therefore determined separate values of $\left\langle E_{\perp}\right\rangle$ for a number of RFA voltages. In order to do so, we normalized all RFA intensity scans to each other at a low value of $V_{R}$, see Fig. 13. After that, for a decrease in normalized RFA signal of $10 \%, 20 \%$, etc. we read the pertaining RFA voltage $V_{R, i}$ at field $B_{1}=0.96 \cdot B_{0}$. At the same signal level, we also read the RFA voltages for the curves taken with reduced $B$ field, and determined a value of $\left\langle E_{\perp}\right\rangle_{i}$ from the linearized $\left\langle E_{\|}\right\rangle$vs. $\alpha$ relation, same as in Fig. 12. We extracted the initial $\left\langle E_{\|}\right\rangle$from Eq. (5) and arrived at the red data points shown in Fig. 14. We overlay the energy distribution determined from 


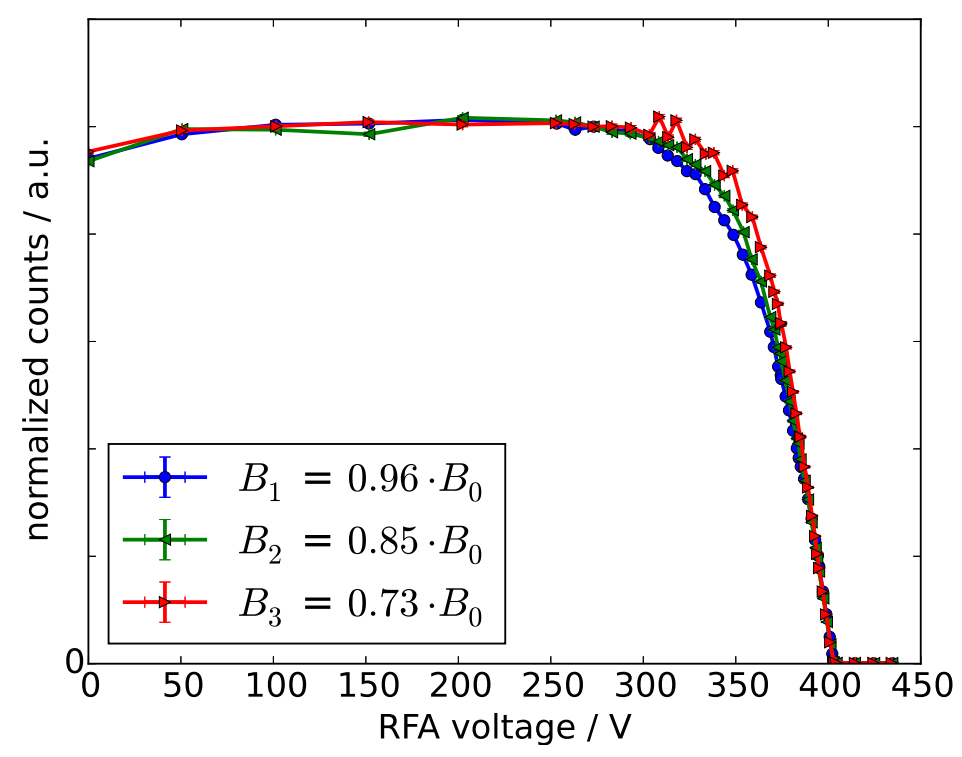

Fig. 13: Normalized beam intensity as a function of retarding voltage $V_{R}$ for the primary beam at $400 \mathrm{eV}$. Values are shown for the original field $B_{1}=0.96 \cdot B_{0}=4.8 \mathrm{mT}$ and two settings using lower fields in the RFA. Traces were normalized to equal intensity at a RFA voltage of $273 \mathrm{eV}$. Lines between the symbols are shown to guide the eye.

the derivative of the smoothed RFA signal in the magnetic field $B_{1}$. In order to tell what is the energy distribution of the primary beam as it arrives at the open beam port we need to estimate the error introduced by our setup. We do this with the help of our numerical simulations. This requires the ratio of the mean transverse energy to the mean longitudinal energy. As was described above, we now have a distribution of mean perpendicular energy with parallel energy instead of one single value. In analogy to beams with a symmetric parallel energy distribution we choose the measurement giving $50 \%$ of the maximum RFA signal intensity which corresponds to an initial mean longitudinal energy of about $375 \mathrm{eV}$ and an initial mean transverse energy of about $18 \mathrm{eV}$, as shown in Fig. 14, and thus a ratio of 0.048. From the simulations, see Fig. 6, we expect the estimate of the energy spread and the mean perpendicular energy to be up to $20 \%$ high for this ratio. At the retarding potential of $375 \mathrm{~V}$, this means the transverse energy estimated to be about $18 \mathrm{eV}$ may actually be closer to $15 \mathrm{eV}$. This is still much higher than anticipated from the design of the source. In addition, in our simulations we observed that only beams with small ratio of mean perpendicular to mean longitudinal energy develop an asymmetric shape when guided non-adiabatically, see Fig. 5. The observation of an asymmetric energy distribution for the primary beam thus suggests that the beam had a 
much smaller ratio than 0.048 of mean transverse to mean longitudinal energy close to the source. The most likely explanation, in our view, is that transport of higher energy beams from the NEPOMUC source to the open beam port is non-adiabatic.

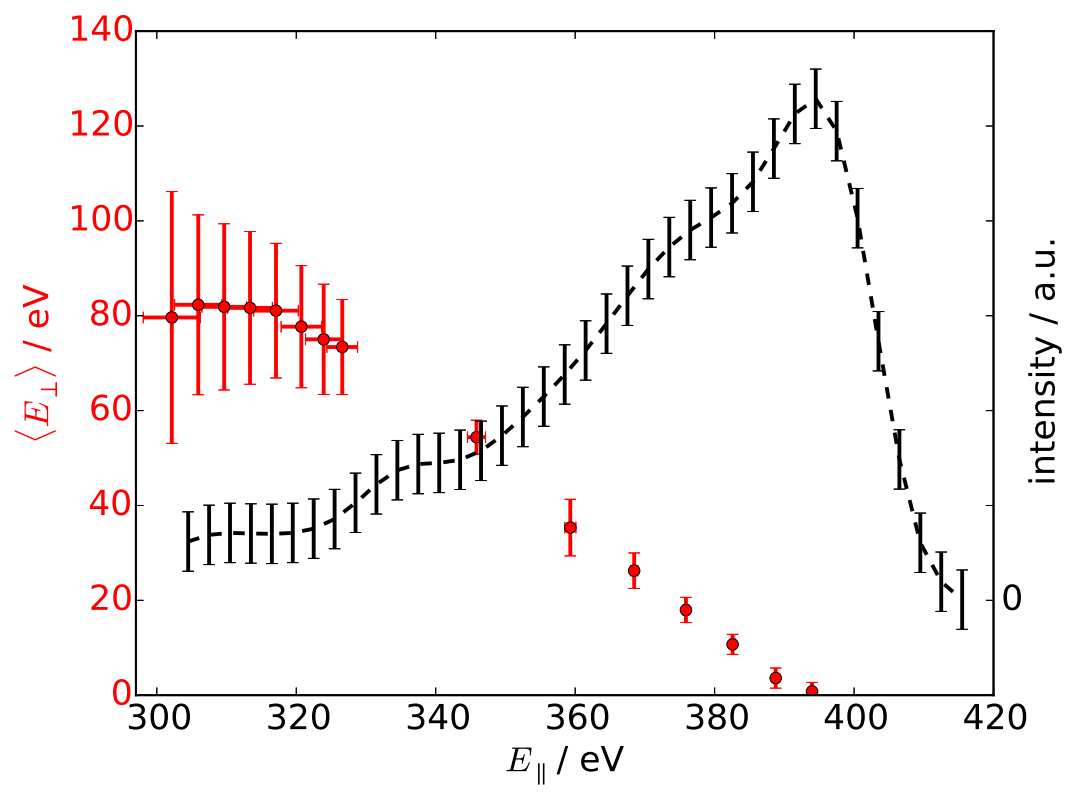

Fig. 14: Distribution of the mean perpendicular energy with respect to the parallel energy for the $400 \mathrm{eV}$ beam (symbols). Overlaid is the energy distribution function obtained from the derivative of the smoothed RFA data measured in the original field of $4.8 \mathrm{mT}$ (error bars connected by a dashed line to guide the eye).

\section{Summary}

In summary, we determined various properties of the primary and remoderated NEPOMUC positron beam at different energies. For the remoderated beam with energies of 5,12 and $22 \mathrm{eV}$ we observed intensities in the range of $(2-6) \cdot 10^{7} e^{+} / \mathrm{s}$, comparable to previous measurements close to the source [11]. The diameter of this beam was found to be less than $4 \mathrm{~mm}$ (FWHM) and independent of the beam energy, which is convenient, e.g., for the investigation of positron injection into a magnetic dipole field [8]. The energy spread of the remoderated beam is found to exceed expectations from thermal broadening perhaps due to surface properties of the remoderation crystal and the remoderator geometry. In general, our results do not indicate that the remoderated beam characteristics are changed during transport from the source to the experiment. 
The primary beam yielded the maximum positron intensity at our set-up, as expected. However, the observed $5 \cdot 10^{8} e^{+/ s}$ is significantly lower than the maximum intensity measured close to the positron source [11]. This result together with the observed energy parameters suggests, in our interpretation, a non-adiabatic transport of positrons from the source to the open beam port. The transport of this beam is further complicated by the rather broad beam diameter of about $14 \mathrm{~mm}$.

In the future, a new beam line will guide positrons to the east extension hall at FRM II. This hall will house a number of experiments, including the pair-plasma experiment. The beam line will meet the requirements for adiabatic transport of high-energy positron beams and thus, in particular for the primary beam, a well-defined energy distribution with smaller energy spread is expected. In the meantime, a decrease in beam energy for the primary beam would help to reduce positron losses in the beam line.

\section{Acknowledgment}

The authors acknowledge contribution of Sebastian Vohburger to setting up the experiment. EVS acknowledges a grant by the Helmholtz Society. This work is based upon experiments performed at the NEPOMUC positron beam facility operated by FRM II at the Heinz Maier-Leibnitz Zentrum (MLZ), Garching, Germany. This work was partially supported by JSPS Grant-in-Aid for Scientific Research 25707043.

\section{References}

[1] Coleman P. Positron beams and their application. World Scientific, 2000.

[2] G. F. Gribakin, J. A. Young, and C. M. Surko. Rev. Mod. Phys., 82: 25572607, 2010.

[3] Nagashima Y. Physics Reports, 545:95-123, 2014.

[4] Alpha Collaboration. Nature Physics, 7:558564, 2011.

[5] AEgIS Collaboration. Eur. Phys. J. D, 68:41, 2014.

[6] J. Mayer, C. Hugenschmidt, and K. Schreckenbach. Phys. Rev. Lett., 105: 207401, 2010.

[7] T. Sunn Pedersen, J. R. Danielson, C. Hugenschmidt, G. Marx, X. Sarasola, F. Schauer, L. Schweikhard, C. M. Surko, and E. Winkler. New J. Phys., 14:035010, 2012.

[8] H. Saitoh, J. Stanja, E. V. Stenson, U. Hergenhahn, H. Niemann, T. Sunn Pedersen, M. R. Stoneking, C. Piochacz, and C. Hugenschmidt. New J. Phys., 17:103038, 2015. 
[9] C. Hugenschmidt, C. Piochacz, M. Reiner, and K. Schreckenbach. New J. Phys, 14:055027, 2012.

[10] C. Hugenschmidt, H. Ceeh, T. Gigl, F. Lippert, C. Piochacz, P. Pikart, M. Reiner, J. Weber, and S. Zimnik. J. Phys.: Conf. Ser., 443:012079, 2013.

[11] C. Hugenschmidt, H. Ceeh, T. Gigl, F. Lippert, C. Piochacz, M. Reiner, K. Schreckenbach, S. Vohburger, J. Weber, and S. Zimnik. J. Phys.: Conf. Ser., 505:012029, 2014.

[12] C. Piochacz, G. Kögel, W. Egger, C. Hugenschmidt, J. Mayer, K. Schreckenbach, P. Sperr, M. Stadlbauer, and G. Dollinger. Appl. Surf. Sci., 255: 98-100, 2008.

[13] C. Hugenschmidt, G. Kögel, R. Repper, K. Schreckenbach, P. Sperr, and W. Triftshäuser. Nucl. Inst. and Meth. in Phys. Res. B, 198:220-229, 2002.

[14] S. Pastuszka, M. Hoppe, D. Kratzmann, D. Schwalm, A. Wolf, A. S. Jaroshevich, S. N. Kosolobov, D. A. Orlov, and A. S. Terekhov. J. Appl. Phys., $88: 6788,2000$.

[15] H. Qin, S. Zhang, J. Xiao, J. Liu, Y. Sun, and W. M. Tang. Phys. Plasmas, 20:084503, 2013.

[16] M. Puska and R. Nieminen. Rev. Mod. Phys., 66:841-97, 1994.

[17] R. G. Wilson. J. Appl. Phys., 37:2261, 1966.

[18] J. Hölzl and F. K. Schulte. Solid Surface Physics. Springer Berlin Heidelberg, 1979.

[19] R. M. Nieminen and C. H. Hodges. Solid State Comm., 18:1115-1118, 1976. 